\title{
Propuesta para la integración de criterios sostenibles en los proyectos de ingeniería civil: un caso práctico
}

\author{
Proposal for the integration of sustainability criteria in civil \\ engineering projects: a case study \\ G. Fernández-Sánchez ${ }^{(*)}$, F. Rodríguez-López $z^{(*)}$
}

\section{RESUMEN}

Se propone en este artículo un sistema de indicadores para la evaluación de la sostenibilidad en los proyectos de infraestructuras lineales desde etapas iniciales (estudio previo de soluciones) para permitir la clasificación de las distintas alternativas según su impacto sobre el medio ambiente, la sociedad y la economía, y lograr así la selección de la solución considerada como más sostenible. Se realiza un breve estudio del estado del conocimiento relativo a la construcción sostenible a nivel internacional y, de acuerdo a las iniciativas existentes, se propone la evaluación de la sostenibilidad de los proyectos de infraestructuras mediante criterios e indicadores. Se aplica este sistema de indicadores a un caso de estudio de una autovía española mediante un análisis multicriterio, de manera que se identifican las limitaciones existentes para la aplicación de criterios sostenibles desde etapas tempranas considerando el ciclo de vida del proyecto, se propone el control y seguimiento de estos indicadores en fases posteriores, así como se sugiere la posible aplicación en la certificación de proyectos.

$113-112$

Palabras clave: sostenibilidad, ingeniería civil, construcción sostenible, indicadores, toma de decisiones

\section{SUMMARY}

In this paper an indicator system is proposed for assessing sustainability in linear infrastructure projects from early stages (previous analysis of alternatives) to allow classification of the different alternatives according to their impact on the environment, society and economy, and thus to select the most sustainable alternative. It is developed a brief study of the state of knowledge on sustainable construction worldwide and, according to existing initiatives, it is proposed the sustainability assessment of infrastructure projects using a set of criteria and indicators. The indicator system is also applied to a case study, a Spanish highway, through a multi-criteria analysis, to identify existing constraints to the implementation of sustainability criteria from early stages considering the project life cycle, taking into account the interest of controlling and monitoring the indicator valuation in later stages and their possible application in the comparison between projects.

Keywords: sustainability, civil engineering, sustainable construction, indicators, decision-making 


\section{INTRODUCCIÓN}

La aplicación inicial de los criterios sostenibles tuvo lugar a escala municipal en la Cumbre de la Tierra de 1992 en Río de Janeiro donde aparece la Agenda 21 y se propone el uso de indicadores para la evaluación, control y vigilancia de las tres dimensiones del desarrollo sostenible.

"Indicators of sustainable development need to be developed to provide solid bases for decision-making at all levels and to contribute to a self-regulating sustainability of integrated environment and development systems" (1).

Posteriormente, el desarrollo sostenible ha ido inundando todas las actividades humanas, incluyendo la construcción. En 1994 apareció por primera vez el concepto de "construcción sostenible" aplicado fundamentalmente al sector de la edificación y, mínimamente, a la ingeniería civil. Comienzan a surgir así nuevos requisitos relacionados con los pilares del desarrollo sostenible (medio ambiente, sociedad y economía) añadidos a los objetivos específicos de los proyectos de construcción (coste, plazo y calidad). Aparece, por tanto, la necesidad de nuevas técnicas y herramientas de gestión que permitan una correcta toma de decisiones desde la etapa de diseño del proyecto hasta su construcción, explotación y mantenimiento considerando su posible deconstrucción, rehabilitación o demolición, pasada su vida útil.

Probablemente no existe un sector con mayor potencial de contribución al desarrollo sostenible que la construcción; el problema radica en la amplitud del sector y en que existen numerosos actores en el ciclo de vida del proyecto (2). Se estima que, aproximadamente, la construcción emplea la mitad de los recursos que el hombre consume de la naturaleza, se considera que el $25 \%$ de los residuos generados en el mundo son residuos de construcción y demolición (3) y que más del $70 \%$ de la energía mundial se mueve alrededor de este sector (4). Es necesario por tanto un equilibro a mayor escala teniendo en cuenta los impactos producidos por la construcción en su entorno, buscando principalmente: la aplicación del Análisis del Ciclo de Vida (ACV) de las infraestructuras y los proyectos, no considerando únicamente la fase de construcción o explotación; aplicar criterios de sostenibilidad desde la fase de planificación y diseño, mediante la colaboración interdisciplinar de todos los actores presentes en el proyecto; y dar especial importancia al entorno social, económico y medioambiental afectado. El problema que surge ahora es cómo aplicar un concepto tan abierto y complejo en un sector tan técnico donde prima la calidad, el coste y el plazo, además de, por supuesto, los requisitos técnicos, del promotor y de cada contexto particular.

\section{ANTECEDENTES}

La gran variedad de campos en los que interviene el ingeniero de caminos hace que se disponga de una gran oportunidad para aplicar criterios de construcción sostenible a sus proyectos, y de éste modo reducir los impactos sobre el medio ambiente a la vez que se proporciona un estado de igualdad, confort y calidad de vida tanto social como económica sin mermar la capacidad de los ecosistemas.

En el campo de la edificación existen numerosas aproximaciones hacia la sostenibilidad, mientras que en los proyectos de infraestructuras su aplicación está resultando mucho más lenta y el uso de criterios sostenibles se basa casi exclusivamente en manuales de buenas prácticas con el entorno.

Según la organización CRISP -Construction and City Related Sustainability Indicators- (5) estos nuevos objetivos sostenibles pueden ser aplicados a la edificación mediante un sistema de indicadores, al igual que ocurría a escala municipal, por las siguientes razones:

- La toma de decisiones en los proyectos de edificación han de ser tomadas en las etapas previas (planificación y diseño) y para ello se necesitan herramientas que permitan calificar el edificio en las diferentes dimensiones de la sostenibilidad.

- La complejidad inherente al concepto de sostenibilidad hace que unos indicadores sencillos simplifiquen el problema de un modo lo más objetivo posible de modo que se pueda estudiar fácilmente el impacto (positivo y negativo) que tendrá el edificio sobre el entorno en el ciclo de vida.

- Los indicadores analizan el edificio mediante criterios que evaluarán los objetivos sostenibles que se quieren alcanzar en las diferentes fases y permite que desde etapas iniciales se pueda gestionar correctamente un proyecto.

De hecho, la aplicación e integración más común del concepto sostenible en la edificación se ha hecho mediante indicadores. Se ha realizado una búsqueda de las herramientas existentes de evaluación de la sostenibilidad de edificios que trabajan por medio de sistemas de indicadores en todo el mundo, obteniendo un total de 74 sistemas de indicadores de edificación (6). De éstas, las más empleadas y conocidas son actualmente LEED (Leadership in Energy and Environmental Design) certificación sostenible gestionada por el USGBC (United States of Green Building Council) basada en una lista de control y que 
dispone ya de 500 proyectos certificados y más de 5.400 proyectos registrados con el fin de certificarse en más de 26 países del mundo (4); SBTool (Sustainable Building Tool 2007) con su aplicación a España denominada VERDE, se basa en criterios-indicadores y es promovida por iiSBE (International Iniciative for a Sustainable Built Environment) en un proyecto que involucró a más de 25 países desde 1998 y que permite que grupos nacionales particularicen la herramienta a las condiciones nacionales (7); BREEAM (Building Research Establishment Environmental Assessment Method) originario del Reino Unido y gestionado por BRE, es el sistema de análisis medioambiental más antiguo que existe $y$, al igual que LEED, se basa en una lista de control; o CASBEE (Comprehensive Assessment System for Building Environmental Efficiency) originario de Japón que comenzó en 2001 y basado en la valoración de impactos utilizando el concepto de eco-eficiencia (relación entre calidad del servicio y cargas ambientales). Actualmente, dos proyectos de investigación buscan la homogeneización de tantos sistemas de evaluación de la sostenibilidad en el sector de la edificación. Por un lado se encuentra LEnSE (Label for Environmental, Social and Economic Buildings) del Sexto Programa Marco de Investigación Europeo finalizado en diciembre de 2007; y por otro, el sello de evaluación sostenible que el World Council of Civil Engineers (WCCE) está Ilevando a cabo.

Sin embargo, la ingeniería civil no ha experimentado un desarrollo o aplicación notable de criterios sostenibles en sus proyectos. La explicación puede encontrarse en la amplia percepción social que se supone tiene la edificación, mientras que en las infraestructuras la existencia de herramientas como la Evaluación de Impacto Ambiental (EIA) y la reciente Evaluación Ambiental Estratégica (EAE) hace que aparezcan como alternativas a las aproximaciones de evaluación de la sostenibilidad (8-9). Sin embargo, con estos modelos se están dejando de lado los pilares sociales y económicos. Como se consensuó en las conclusiones del $\mathrm{V}$ Congreso Nacional de la Ingeniería Civil "Desarrollo y Sostenibilidad en el Marco de la Ingeniería" (10), era precisa una concienciación sobre la necesidad de aplicar los criterios sostenibles en todas las actividades de la sociedad, incluidas las relativas a los proyectos de infraestructuras. En esta misma línea se está trabajando en el actual Grupo de Trabajo "Infraestructuras, Transporte y Sostenibilidad" del Conama 10.

En los últimos años han comenzado a surgir modelos de evaluación sostenible basados en sistemas de indicadores para los proyectos de ingeniería civil, como el sistema SUSAIP, SUStainability Appraisal in Infrastructure
Projects (11) aplicable a puentes y viaductos; el modelo TSI, Technical Sustainability Index, propuesto por Dasgupta y Tam (12) para su aplicación en proyectos de infraestructuras en Canadá, especialmente infraestructuras eléctricas; sucesivas aplicaciones del sistema LEED de edificios a los proyectos de carreteras en EEUU $(13,14)$ con las complicaciones que esto conlleva; o el nuevo ICES (Índice de Contribución de las Estructuras a la Sostenibilidad) de la Instrucción EHE española (15) válido para la evaluación de estructuras de hormigón en edificación y obra civil. En esta tipología de proyectos ha surgido también en el Reino Unido, fruto de la colaboración entre Institution of Civil Engineers (ICE) y el Departamento de Medioambiente, Transporte y las Regiones del Reino Unido un modelo de evaluación sostenible (CEEQual) basado en una lista de control de doce dimensiones relacionadas con los pilares social y ambiental, y aplicado recientemente a las obras geotécnicas y de terraplenado del ferrocarril de Londres (16).

Se debe destacar, además, la existencia del Comité Técnico ISO/TC 59/SC 17 Building Construction/Sustainability in building construction, dedicado a la construcción en general y estructurado en cinco grupos de trabajo, el último de los cuales se dedica a los proyectos de ingeniería civil de una manera específica. En el año 2009 se encontraba desarrollando criterios de sostenibilidad para alguno de los proyectos de ingeniería civil, concretamente para la tipología de presas con el borrador "Sustainability indicators for dams", todavía en etapas muy tempranas y presentado en España el 30 de marzo de 2009. Este grupo de trabajo, será en un futuro cercano un auténtico referente en la temática que aquí se expone.

Con las distintas aproximaciones existentes hacia un modelo de evaluación sostenible, la construcción sostenible busca fundamentalmente los siguientes objetivos:

- Minimizar los recursos utilizados (materiales, agua y energía) mediante una correcta gestión de materias primas, reutilización y reciclaje.

- Minimizar los residuos generados fundamentada en la gestión de residuos: materiales reutilizables, reciclables y/o valorables energéticamente.

- Minimizar el impacto de las emisiones directas e indirectas al agua, a la atmósfera y a la tierra, con una especial importancia de las emisiones de Gases Efecto Invernadero (GEI) y su relación con el cambio climático.

- Innovar y usar energías renovables y materiales de bajo impacto ambiental.

- Analizar y minimizar los impactos sobre el entorno social, no solo sobre los usuarios directos del proyecto sino también los usuarios indirectos. Accesibilidad. 


\section{Metodología propuesta.}

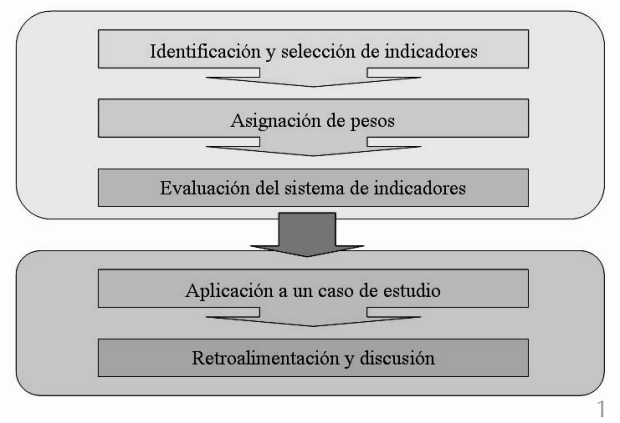

- Estudiar la relación coste/beneficio de las infraestructuras en su ciclo de vida, tratando de que el valor sea el menor posible (mayor beneficio y menor coste).

- Disminuir el coste en el ciclo de vida del proyecto (Coste en el Ciclo de Vida).

- Aumentar la funcionalidad y la flexibilidad del proyecto para futuros cambios o modificaciones.

- Minimizar el impacto sobre la biodiversidad y el entorno medioambiental.

Y en búsqueda de estos objetivos se necesitan modelos prácticos y sencillos que permitan su aplicación a los estudios previos de soluciones o anteproyectos para la selección de las alternativas más sostenibles, así como de índices que engloben la sostenibilidad de un proyecto de construcción para su posible certificación, como se hace actualmente en la edificación.

Parece claro pues que el modelo de evaluación sostenible a aplicar en los proyectos de ingeniería civil como ayuda para la toma de decisiones, aprovechando las lecciones aprendidas de otros sectores tanto a nivel local en los municipios como en el sector de la edificación, es sensato llevarlo a cabo mediante indicadores de sostenibilidad que permitan el análisis de los objetivos sostenibles por separado, marcar tendencias y lograr un control y monitorización sobre dichos objetivos en el tiempo.

\section{OBJETIVOS Y METODOLOGÍA}

El objetivo principal de este artículo es la propuesta de un sistema de indicadores de sostenibilidad, con un modo de evaluación de cada criterio (cualitativa y/o cuantitativamente) y su integración en los proyectos de infraestructuras lineales donde no existen iniciativas para la evaluación de la sostenibilidad, proponiendo su integración desde las fases iniciales del proyecto.

Así, la metodología propuesta para la creación de un sistema de indicadores de sostenibilidad y su posterior aplicación a los proyectos de infraestructuras aparece reflejada en el siguiente esquema (Figura 1). Se comenzará con una identificación y selección de indi- cadores concediendo especial importancia a la participación de todos los agentes involucrados en los proyectos de infraestructuras, asignando a cada criterio y dimensión un peso de modo orientativo (modificables según el promotor o administración), y se proponen sistemas de evaluación de los distintos indicadores tanto a nivel individual (criterio a criterio) como conjuntamente. Por último, se aplica a un caso de estudio en las etapas iniciales (estudio previo de soluciones) y una discusión y conclusiones de la propuesta.

\section{SISTEMA DE INDICADORES DE SOSTENIBILIDAD PROPUESTO}

Partiendo de la metodología sugerida en el apartado anterior y tomando como hipótesis la consideración de la sostenibilidad como una oportunidad de mejora para el proyecto (riesgo positivo), se han aplicado los estándares de gestión de riesgos y oportunidades con objeto de lograr una identificación y selección de indicadores válidos para la evaluación de la sostenibilidad en el ámbito de las infraestructuras lineales en España desde las etapas iniciales (17). Estos indicadores han sido seleccionados mediante ocho técnicas de identificación de oportunidades (revisión de la documentación, legislación nacional, encuestas, entrevistas, reunión de expertos, técnicas de diagramación, listas de control y comparación con otras áreas similares), primando la participación de todos los involucrados en un proyecto de infraestructuras: la administración-promotor, los centros de investigación y universidades, las ingenierías y consultoras, los fabricantes y distribuidores, las constructoras, los usuarios, las empresas de mantenimiento y explotación, los ecologistas, los expertos en evaluaciones de impacto ambiental y, por último, distintos organismos dedicados activamente a las aplicaciones prácticas de la sostenibilidad, como el Observatorio de la Sostenibilidad en España (OSE) y el Observatorio de la Aviación Sostenible (OBSA). El objetivo principal en la identificación de estos indicadores de sostenibilidad ha sido el intento de gestión multidisciplinar de la sostenibilidad para lograr un consenso en la selección definitiva del sistema de indicadores.

Tras una identificación inicial de, aproximadamente, 400 indicadores potencialmente aplicables, se procedió a una agrupación de los mismos siguiendo el estándar ISO 219291 (18) determinando un total de 60 macroindicadores. El conjunto final de indicadores quedó cerrado reuniendo a un pequeño panel de expertos multidisciplinar donde se trató de lograr un consenso mediante la aplicación del modelo AHP (Analytic Hierarchy Process) comparando por pares las variables identificadas. La Tabla 1 muestra la propuesta de los 30 indicadores finalmente seleccionados con la ponderación resultado de la aplicación del 
método AHP. Lógicamente, esta ponderación será siempre variable por el centro decisor que maneje el proyecto en cada momento de acuerdo a las preferencias y al contexto dado, siendo estos pesos una guía útil para su ponderación. De hecho, es posible que uno de los indicadores sea seleccionado como de obligado cumplimiento, mientras que otros se desestimen o se consideren inoportunos según el caso que se trate.

Las diferentes alternativas existentes en un anteproyecto de una infraestructura lineal se pueden evaluar ahora atendiendo al sistema de indicadores propuesto para la selección de la mejor solución desde el punto de vista de la sostenibilidad integral del proyecto. El modelo que se propone es un análisis multicriterio MCDA (Multi Criteria Decision Aid), con las variables de sostenibilidad mostradas en la Tabla 1, que permita discernir las soluciones más sostenibles entre aquellas existentes y la posible creación de nuevos escenarios o soluciones que optimicen alguno o varios de estos indicadores. Igualmente, el sistema de indicadores puede ser utilizado para optimizar la alternativa finalmente escogida con el objetivo de certificar el grado de sostenibilidad alcanzado de un proyecto de construcción y aportar un valor adicional al proyecto. En la siguiente figura (Figura 2) se muestran las dos posibles aplicaciones de este modelo de gestión sostenible para los proyectos de infraestructuras.

Según este esquema, se propone la utilización del sistema de indicadores propuesto desde la fase inicial de un proyecto (estudio previo) para analizar las distintas alternativas existentes según sus materiales empleados, la gestión de residuos propuesta, las posibilidades de trazados, los costes en el ciclo de vida, los consumos, los diferentes diseños e incluso la generación de nuevas alternativas atendiendo a las variables de sostenibilidad mencionadas. Las posibilidades de evaluación del proyecto son de tipo cualitativo y de tipo cuantitativo. Lógicamente, parece más fiable, segura y técnica la segunda opción. Existe una gran controversia entre la valoración cualitativa o cuantitativa de los indicadores de sostenibilidad en general. Autores como Bell y Morse (19) sostienen y discuten la ventaja de tomar un enfoque holístico y cualitativo mejor que el esfuerzo de tomar medidas de una manera cuantitativa y estricta. Aguado (20) llega a ejemplificar cómo las opiniones de expertos mediante un análisis cualitativo (opinión de expertos usando el modelo AHP) pueden aportar valores muy próximos a los valores cuantitativos sin la necesidad de tomar medidas y valores exactos. En el caso de un indicador indiscutible como es el de emisiones de Gases Efecto Invernadero (GEI ó $\left.\mathrm{CO}_{2 \mathrm{eq}}\right)$, se está tratando de desarrollar en

\section{Tabla 1}

Sistema de indicadores propuesto para la ayuda a la toma de decisiones en los proyectos de infraestructuras lineales en España desarrollado a partir de Fernández-Sánchez y Rodríguez-López (17)

\begin{tabular}{|c|c|c|c|}
\hline Dimensiones & Macro-indicadores & Peso & $\begin{array}{c}\text { Peso } \\
\text { Dimensiones }\end{array}$ \\
\hline \multirow{10}{*}{ Medioambiente } & Gestión de residuos & $4,96 \%$ & \multirow{10}{*}{$35,02 \%$} \\
\hline & Huella ecológica & $4,78 \%$ & \\
\hline & Emisiones de $\mathrm{CO}_{2 \mathrm{eq}}$ & $4,72 \%$ & \\
\hline & Consumo de materiales & $4,22 \%$ & \\
\hline & Protección del recurso agua & $3,45 \%$ & \\
\hline & Efecto barrera & $3,00 \%$ & \\
\hline & Protección de la biodiversidad & $2,75 \%$ & \\
\hline & Gestión medioambiental & $2,45 \%$ & \\
\hline & Valor ecológico del suelo & $2,43 \%$ & \\
\hline & Contaminación sonora - Ruido & $2,26 \%$ & \\
\hline \multirow{10}{*}{ Sociedad } & Seguridad y Salud & $4,68 \%$ & \multirow{10}{*}{$31,00 \%$} \\
\hline & Necesidad social y urgencia del proyecto & $4,58 \%$ & \\
\hline & Interés general y social del proyecto & $3,60 \%$ & \\
\hline & $\begin{array}{l}\text { Gestión de riesgos ante desastres (inun- } \\
\text { daciones, terremotos) }\end{array}$ & $3,45 \%$ & \\
\hline & Participación pública & $3,15 \%$ & \\
\hline & $\begin{array}{l}\text { Accesibilidad para la biodiversidad } \\
\text { humana }\end{array}$ & $2,75 \%$ & \\
\hline & Respeto a las costumbres locales & $2,60 \%$ & \\
\hline & Uso de materiales regionales & $2,13 \%$ & \\
\hline & Impacto visual & $2,13 \%$ & \\
\hline & Funcionalidad y flexibilidad & $1,93 \%$ & \\
\hline \multirow{10}{*}{ Economía } & Consumo energético & $5,32 \%$ & \multirow{10}{*}{$33,98 \%$} \\
\hline & Coste en el Ciclo de Vida (LCC) & $4,52 \%$ & \\
\hline & Uso de energías renovables & $4,19 \%$ & \\
\hline & Relación coste / beneficio & $3,94 \%$ & \\
\hline & $\begin{array}{l}\text { Adaptación y vulnerabilidad al cambio } \\
\text { climático y ambiental }\end{array}$ & $3,34 \%$ & \\
\hline & Diseño para el desmontaje (DfD) & $3,26 \%$ & \\
\hline & $\begin{array}{l}\text { Gestión del proyecto y gestión } \\
\text { estratégica }\end{array}$ & $2,89 \%$ & \\
\hline & Elementos innovadores & $2,49 \%$ & \\
\hline & Gastos ocasionados a los usuarios & $2,30 \%$ & \\
\hline & $\begin{array}{l}\text { Incremento del valor económico } \\
\text { del entorno }\end{array}$ & $1,73 \%$ & \\
\hline
\end{tabular}

varias investigaciones modelos para cuantificar la huella de carbono de los proyectos de construcción con una aproximación de ciclo de vida. Del mismo modo ocurre con el resto de indicadores seleccionados en el sistema propuesto como el consumo energético en el ciclo de vida, el cálculo de la huella ecológica, el análisis del coste en el ciclo de vida, etc.

2. Propuesta para la toma de decisiones y la certificación según los objetivos de la construcción sostenible.

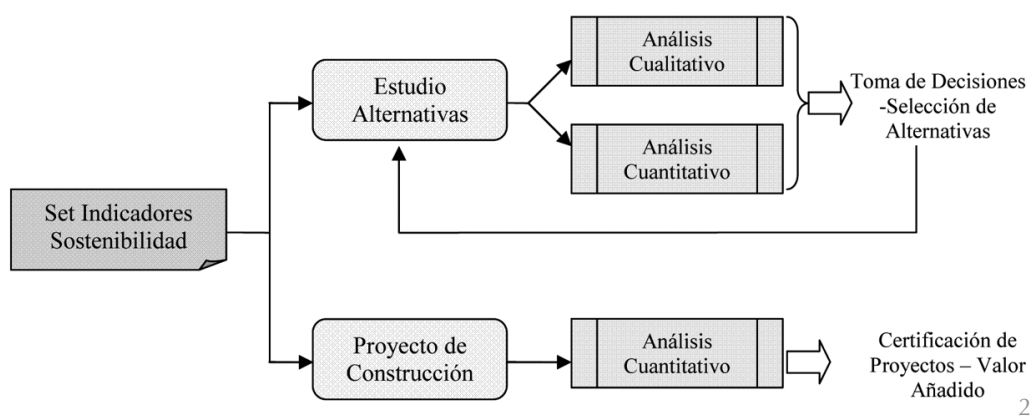




\begin{tabular}{|c|c|c|c|c|c|c|c|c|c|c|c|c|c|c|c|c|c|c|c|c|}
\hline 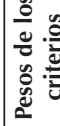 & $\begin{array}{l}0 \\
\text { م. } \\
0\end{array}$ & 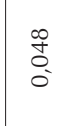 & $\left|\begin{array}{l}f \\
0 \\
0 \\
0\end{array}\right|$ & $\begin{array}{l}\text { f } \\
0 \\
0\end{array}$ & $\begin{array}{l}\text { 足 } \\
0 \\
0\end{array}$ & $\begin{array}{l}0 \\
\stackrel{0}{0} \\
0\end{array}$ & $\begin{array}{l}\hat{a} \\
\text { ò }\end{array}$ & $\begin{array}{l}\stackrel{+}{\Delta} \\
\stackrel{0}{0}\end{array}$ & \begin{tabular}{l}
\multirow{2}{*}{} \\
0 \\
0
\end{tabular} & 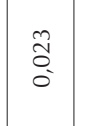 & $\begin{array}{l}\text { ô } \\
\text { Oे }\end{array}$ & $\begin{array}{l}\text { Jư } \\
0 \\
0\end{array}$ & $\begin{array}{l}0 \\
: \\
: \\
0\end{array}$ & $\begin{array}{l}\text { 峁 } \\
0 \\
0\end{array}$ & $\begin{array}{l}\overline{0} \\
0 \\
0\end{array}$ & $\begin{array}{l}\hat{a} \\
\text { ò } \\
0\end{array}$ & $\begin{array}{l}\text { I̊ } \\
\text { o. }\end{array}$ & $\bar{\delta}$ & $\begin{array}{l}\bar{\delta} \\
\text { ¿. }\end{array}$ & $\begin{array}{l}\sigma \\
\bar{\delta} \\
0\end{array}$ \\
\hline 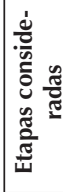 & 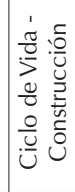 & 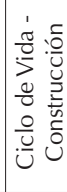 & 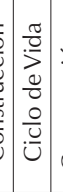 & 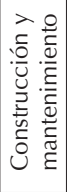 & 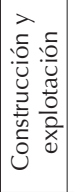 & 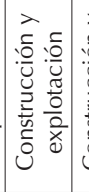 & 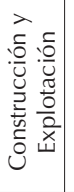 & $\begin{array}{l}\frac{\pi}{0} \\
\frac{0}{2} \\
\frac{0}{0} \\
\frac{0}{0} \\
0\end{array}$ & 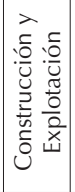 & 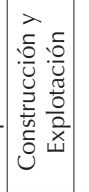 & 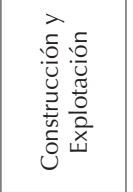 & 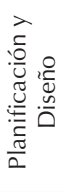 & 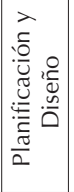 & 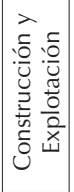 & 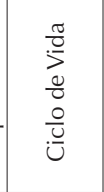 & 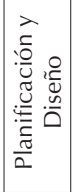 & 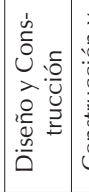 & 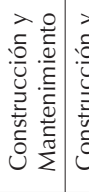 & 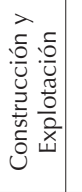 & $\begin{array}{l}\frac{\pi}{0} \\
\dot{0} \\
\frac{0}{0} \\
\frac{0}{0} \\
0\end{array}$ \\
\hline $\begin{array}{l}\frac{0}{\bar{E}} \\
\frac{\bar{E}}{\pi} \\
\frac{0}{0} \\
\frac{0}{0} \\
\frac{0}{2}\end{array}$ & 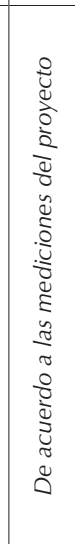 & 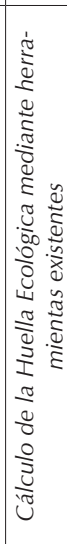 & 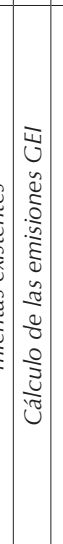 & 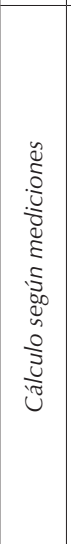 & 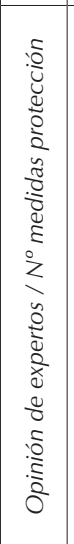 & 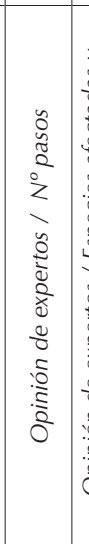 & 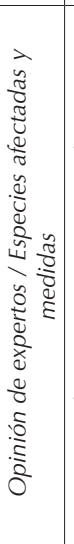 & 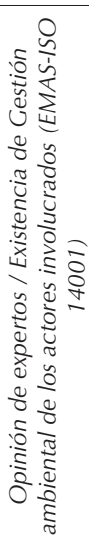 & 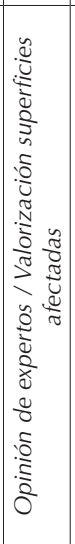 & 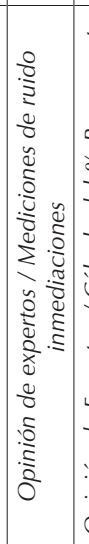 & 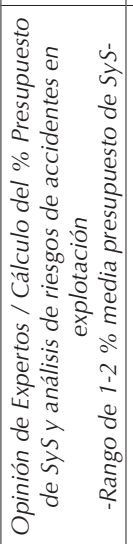 & 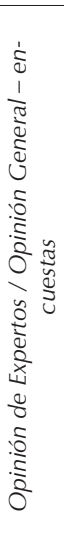 & 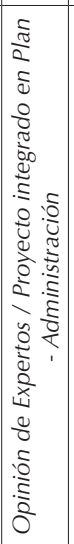 & 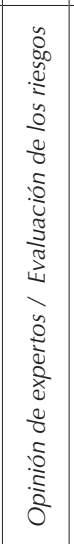 & 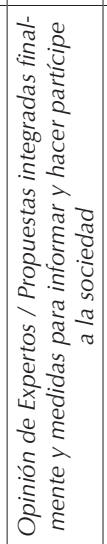 & 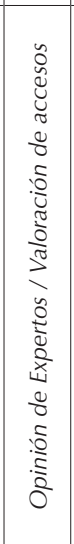 & 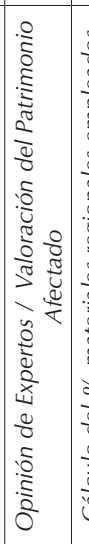 & 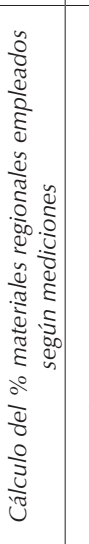 & 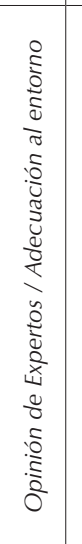 & 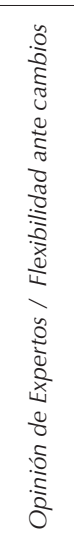 \\
\hline 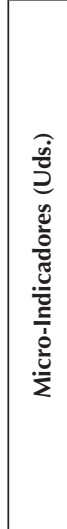 & 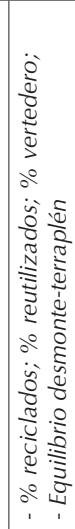 & $\stackrel{\pi}{!}$ & : & 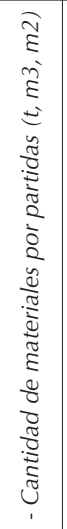 & 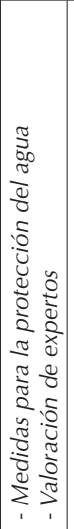 & 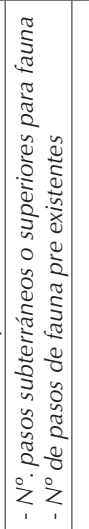 & 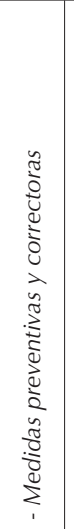 & 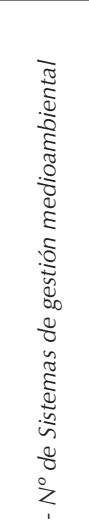 & 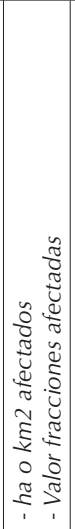 & 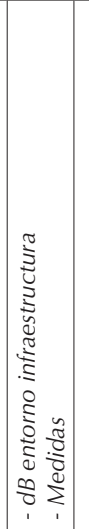 & 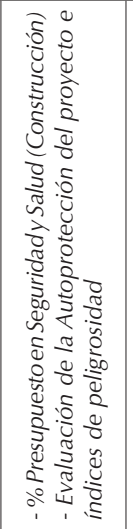 & 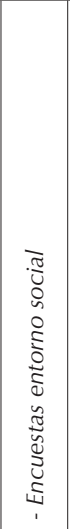 & 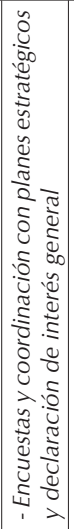 & 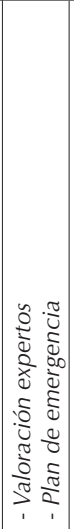 & 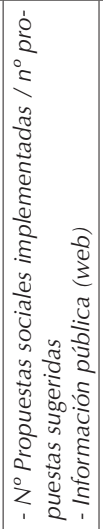 & 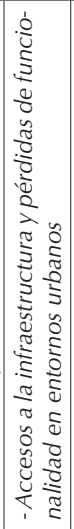 & 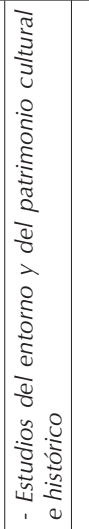 & 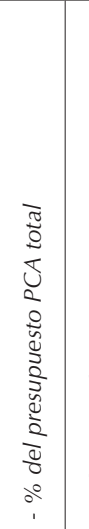 & 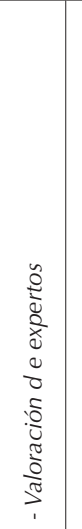 & 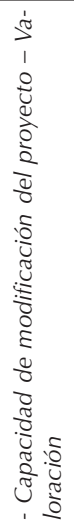 \\
\hline 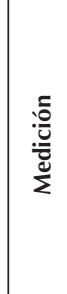 & 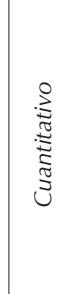 & 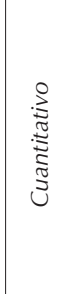 & 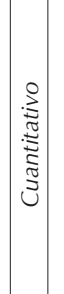 & 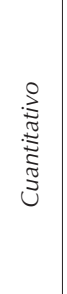 & 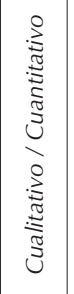 & 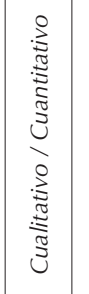 & 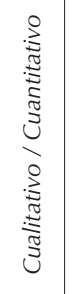 & 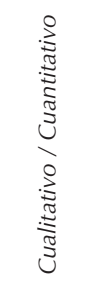 & 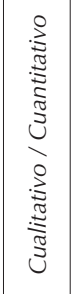 & 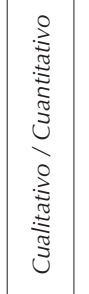 & 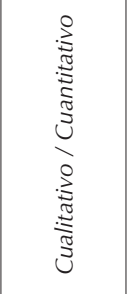 & 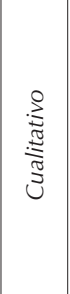 & 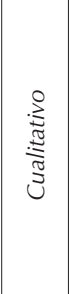 & 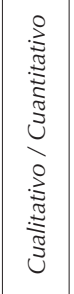 & 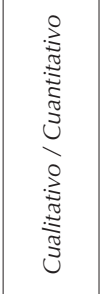 & 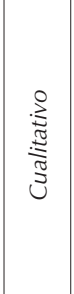 & 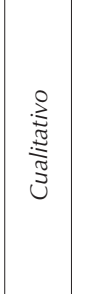 & 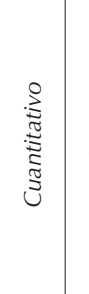 & 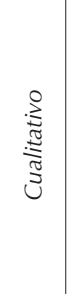 & 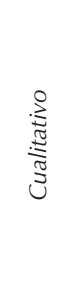 \\
\hline 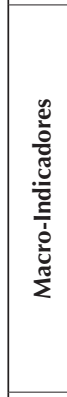 & 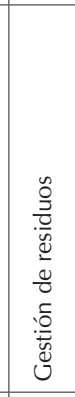 & 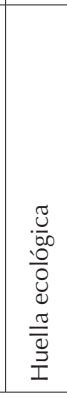 & 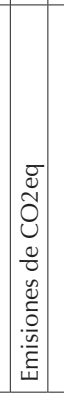 & 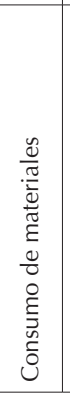 & 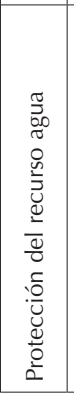 & 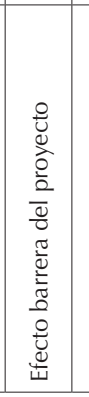 & 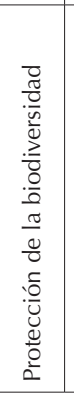 & 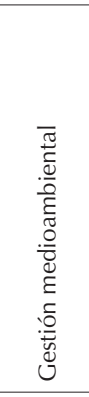 & 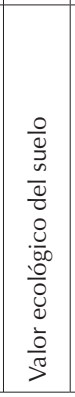 & 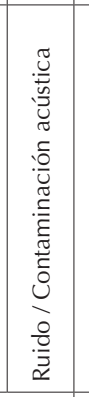 & 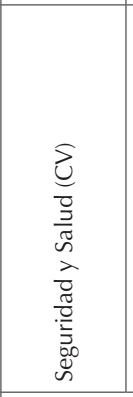 & 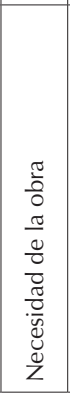 & 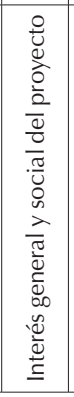 & 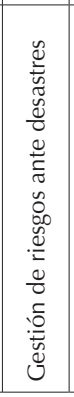 & 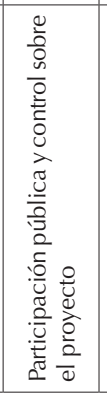 & 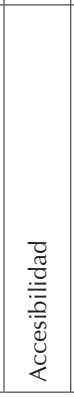 & 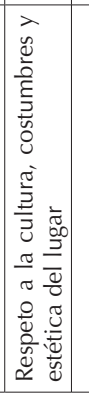 & 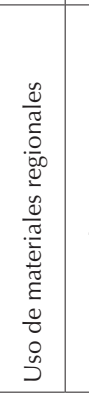 & 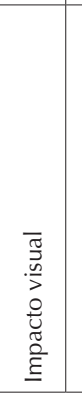 & 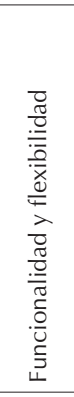 \\
\hline 这 & \multicolumn{10}{|c|}{ 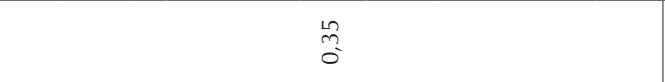 } & \multicolumn{10}{|c|}{$\begin{array}{l}\bar{m} \\
0\end{array}$} \\
\hline 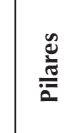 & \multicolumn{10}{|c|}{ 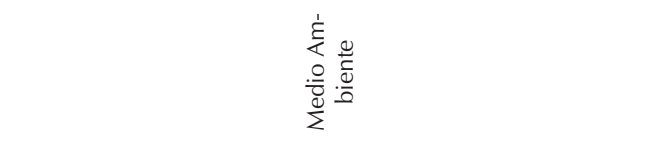 } & \multicolumn{10}{|c|}{ 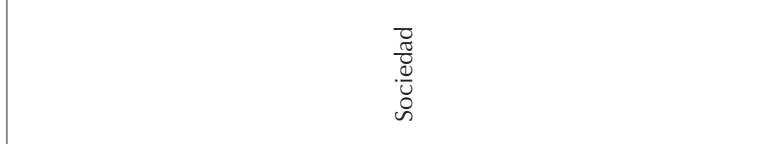 } \\
\hline
\end{tabular}




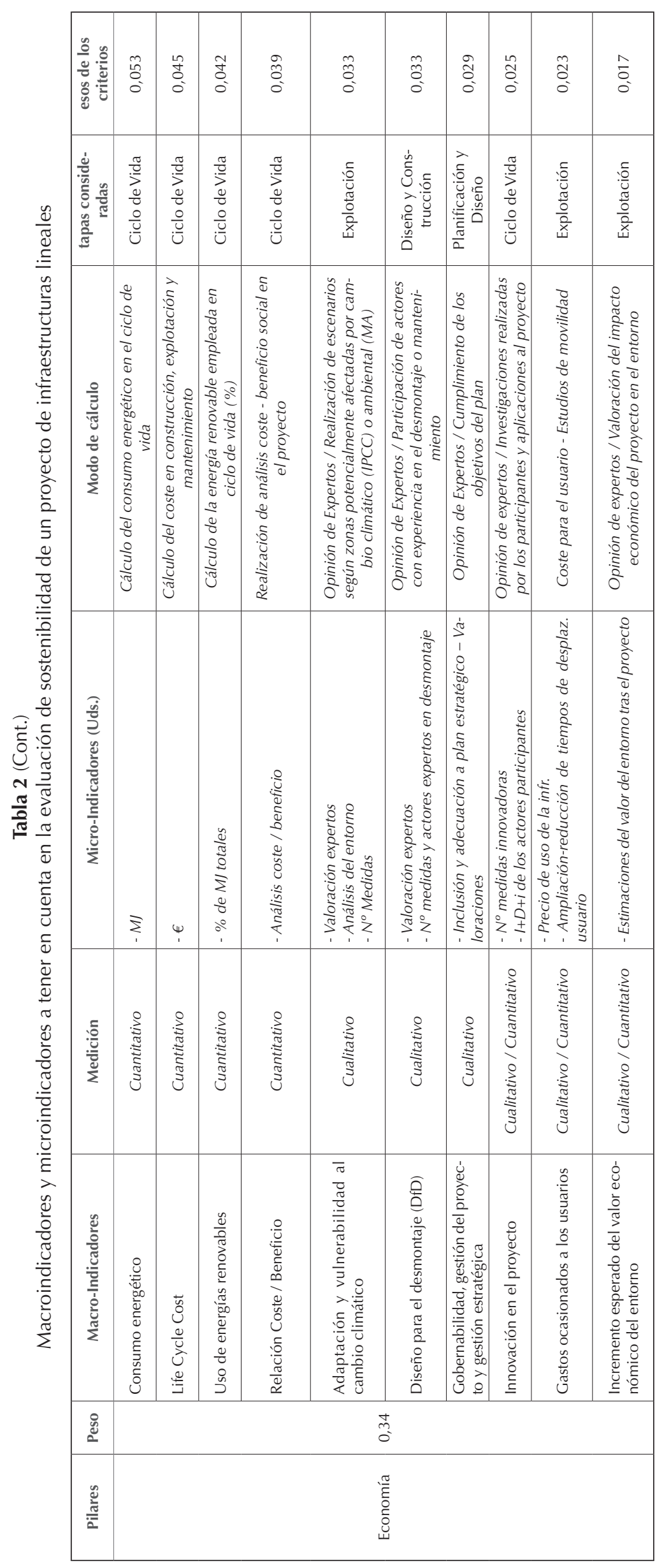

Si bien estos estudios son muy positivos para lograr herramientas precisas y que permiten cuantificar con diferentes fiabilidades los valores totales de cada indicador (análisis cuantitativo), el esfuerzo para su evaluación y cuantificación parece que resulta muy costoso en términos económicos y de tiempo. Por ello, consideramos que en las etapas previas de un proyecto de infraestructuras, en los casos de toma de decisiones entre distintas alternativas, puede resultar mucho más sencillo un análisis cualitativo o cuantitativo basado en datos estimados (predimensionamiento) que permita una rápida aproximación a la situación de cada solución desde el punto de vista de la sostenibilidad. Sin embargo, sí que consideramos necesaria la cuantificación de cada una de las variables en la fase final del proyecto donde lo que se busca es la optimización posible de la solución escogida y su posible certificación, aunque de momento es un proceso que debe tomar su tiempo no sólo para cuantificar cada variable sino también para lograr establecer unos parámetros y unos límites entre lo mínimo aceptable y lo máximo admisible.

Para lograr un mayor desarrollo de los 30 macro-indicadores expuestos, se desglosa a continuación (Tabla 2) los micro-indicadores incluidos en cada una de las variables que deben ser tenidos en cuenta. El análisis multicriterio de estas variables puede desarrollarse de manera integral para todos los criterios propuestos o bien, realizarlo por pilares de sostenibilidad, dimensiones o impactos. Es aquí donde mayor incertidumbre tiene lugar, pues se suman las mediciones de todos los indicadores con unidades distintas (toneladas, $\mathrm{m}^{3}$ o valoración de expertos), y se integran en uno o varios índices de sostenibilidad que obliga a normalizar cada criterio en un rango lógico.

\section{CASO PRÁCTICO}

Este sistema de indicadores se ha aplicado a un caso de estudio de una autovía mediante el análisis de un estudio previo de soluciones, pues es aquí donde se considera que existe una mayor flexibilidad en el proyecto, los cambios resultan menos costosos y la capacidad de aportación por parte de los distintos actores es más alta. De este modo, el caso escogido es la Autovía Medinaceli-Soria en las dos fases del estudio informativo. El trazado de la carretera se divide en cuatro tramos. Los tres primeros disponen de las siguientes alternativas:

- Alternativa 1, con una longitud de 65.079 metros, como nueva construcción de autovía siguiendo un corredor predefinido.

- Alternativa 2, con una longitud de 65.554 metros también como autovía de nueva construcción. 
. Resultados de aplicación del Sistema de Indicadores de Sostenibilidad propuesto al caso práctico seleccionado por tramos y alternativas.
- Alternativa 3, con la idea de reutilizar la actual N-111 y proceder a su duplicación de calzada. Esta alternativa sigue el mismo corredor que la alternativa 1.

En el tramo IV, como acceso final a Soria, se propone tanto el acceso directo al sur de Soria como la unión con la autovía preexistente A-800.

La aplicación del sistema de indicadores de sostenibilidad propuesto se ha realizado de acuerdo a las mediciones y los datos aportados en el estudio informativo, en general predimensionados y estimados, así como la valoración emitida por los autores en el proyecto en la valoración de los criterios cualitativos. Todas las valoraciones de cada indicador han sido normalizadas en un rango de 0 a1 como suele ser habitual en España (21), de manera que sea posible la aplicación de un análisis multicriterio y así obtener un índice de sostenibilidad tanto global (del conjunto de indicadores) como parcial (de cada dimensión: medioambiental, social y económica). El análisis multicriterio se ha realizado siguiendo el algoritmo Pres (22, 23) basado en la comparación por pares de alternativas para cada criterio según la dominación de una solución frente al resto de acuerdo a los valores normalizados y su peso relativo. Además de alguna complejidad encontrada a la hora de aplicar el sistema de indicadores propuesto al proyecto caso de estudio, que se comentará en el apartado de conclusiones, se muestran a continuación los resultados obtenidos para cada tramo y alternativa según las dimensiones del desarrollo sostenible en un diagrama de araña así como definiendo cada alternativa con un índice de sostenibilidad obtenido en cada tramo (Figura 3).

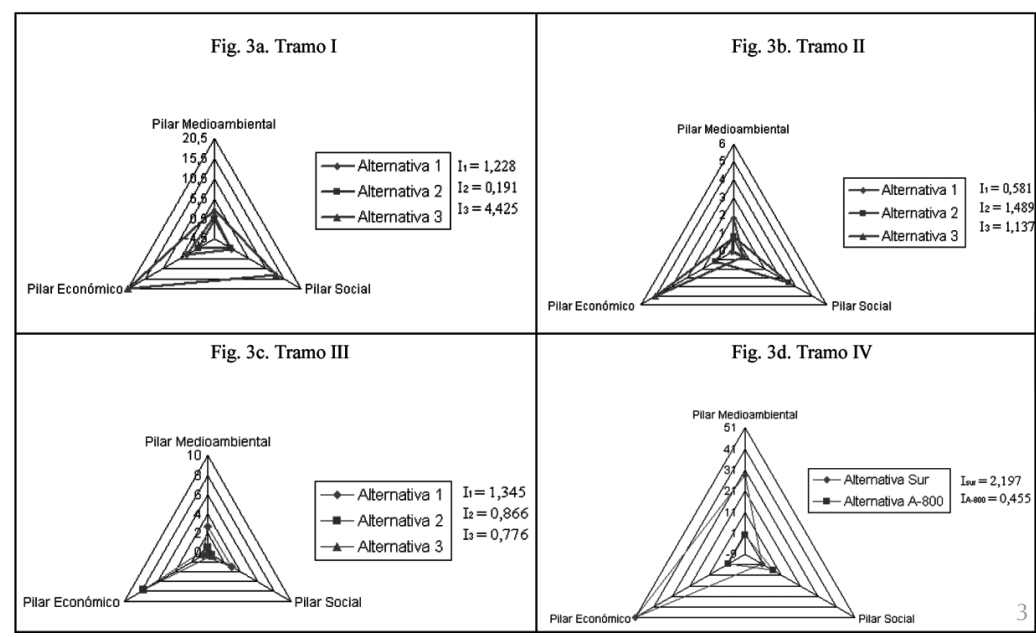

De este modo, se pueden obtener aquellas soluciones más sostenibles desde el punto de vista de los criterios seleccionados. En el primer tramo, la alternativa 3 (duplicación de calzada) es claramente superior tanto en el índice de sostenibilidad $\left(I_{3}=4,425\right)$ como en las dimensiones medioambiental, social y eco- nómica. Lo mismo sucede en el tramo IV donde la primera alternativa destaca sobre la segunda (A-800). Sin embargo, en el resto de tramos las alternativas varían según a qué criterios se de mayor importancia. El tramo II resulta un caso particular donde las diferentes alternativas tienen una mayor valoración según se atienda a la dimensión medioambiental, económica o social. Según la asignación de pesos aquí propuesta, los índices de sostenibilidad de las alternativas analizadas resultan muy similares aunque con diferentes valores que permitirían la selección de alternativas según estos criterios. En estos casos, el centro decisor podrá estudiar en profundidad cuales son aquellos indicadores o dimensiones que tienen mayor importancia en el proyecto o aceptar la valoración obtenida mediante esta propuesta.

Como se muestra en la Tabla 3, no se han podido evaluar todas las etapas de los indicadores en el ciclo de vida del proyecto debido en ocasiones a la falta de información en el estudio centrándose en los aspectos de la fase de construcción y ejecución, así como por la falta de datos o herramientas científicas que permitan una estimación y valoración de los distintos indicadores en el ciclo de vida de la infraestructura. Aparecen sombreadas aquellas fases que han podido ser estimadas de cada indicador.

\section{CONCLUSIONES}

Actualmente, el reto más importante de la sostenibilidad es la interiorización de la necesidad de un reequilibrio entre el entorno y la actividad que realizamos en él. En la ingeniería civil, este nuevo equilibro puede lograrse mediante la adopción de un sistema fácilmente aplicable y flexible desde las primeras fases del proyecto, de manera que desde la generación y selección de alternativas se permita la integración de indicadores con el fin de evaluar y controlar las variables consideradas más importantes dentro de una construcción sostenible.

El sistema de indicadores propuesto se ha aplicado ante una autovía española, actualmente en construcción, en la fase de selección de alternativas con la limitación de que los datos con los que se trabajan en esta etapa han sido generalmente estimados (predimensionamiento); pero también es donde se tiene una mayor capacidad de cambio, a menor coste y con mayor posibilidad de participación. A lo largo del ciclo de vida del proyecto (construcción, explotación y mantenimiento), se propone hacer un control de los distintos indicadores para lograr un acercamiento a las evaluaciones cuantitativas del proyecto. Se debe señalar que en estas fases la capacidad de cambio será ya menor, y la tarea consistirá en la optimización o monitorización de la solución adoptada pero no generar ya cambios sustanciales en ella. 
Tabla 3

Fases del ciclo del proyecto que han podido ser calculadas o estimadas

\begin{tabular}{|c|c|c|c|c|c|c|}
\hline \multirow{2}{*}{ Dimensiones } & \multirow{2}{*}{ Macro-Indicadores } & \multicolumn{5}{|c|}{ Etapas consideradas en el macro-indicador $=(\mathrm{X})$} \\
\hline & & $\begin{array}{c}\text { Planificación / } \\
\text { Diseño }\end{array}$ & Construcción & $\begin{array}{c}\text { Uso y } \\
\text { Explotación }\end{array}$ & Mantenimiento & Fin de vida \\
\hline \multirow{10}{*}{$\begin{array}{c}\text { Medio } \\
\text { Ambiente }\end{array}$} & Gestión de residuos & - & $x$ & $\mathrm{x}$ & $\bar{x}$ & $\bar{x}$ \\
\hline & \begin{tabular}{|l} 
Huella ecológica \\
\end{tabular} & - & $x$ & $x$ & $x$ & $x$ \\
\hline & Emisiones de $\mathrm{CO} 2 \mathrm{eq}$ & - & $\mathrm{x}$ & $\mathrm{x}$ & $x$ & $\mathrm{x}$ \\
\hline & Consumo de materiales & - & $\mathrm{x}$ & - & $x$ & - \\
\hline & Protección del recurso agua & - & $\mathrm{x}$ & $\mathrm{x}$ & $\mathrm{x}$ & $\mathrm{x}$ \\
\hline & Efecto barrera del proyecto & - & $x$ & $x$ & $x$ & - \\
\hline & Protección biodiversidad & - & $\mathrm{x}$ & $x$ & $\mathrm{x}$ & - \\
\hline & \begin{tabular}{|l|} 
Gestión medioambiental \\
\end{tabular} & $\mathrm{x}$ & $x$ & $x$ & $\mathrm{x}$ & $x$ \\
\hline & Valor ecológico del suelo & - & $\mathrm{x}$ & $\mathrm{x}$ & $x$ & - \\
\hline & Ruido / Cont. acústica & - & $x$ & $\mathrm{x}$ & $x$ & $x$ \\
\hline \multirow{10}{*}{ Sociedad } & Seguridad y Salud (CV) & $x$ & $x$ & $\mathrm{x}$ & $x$ & $\mathrm{x}$ \\
\hline & \begin{tabular}{|l|} 
Necesidad de la obra \\
\end{tabular} & - & - & $x$ & - & - \\
\hline & Interés general y social & - & - & $x$ & - & - \\
\hline & $\begin{array}{l}\text { Gestión de riesgos ante } \\
\text { desastres }\end{array}$ & - & $x$ & $x$ & $\mathrm{x}$ & - \\
\hline & Participación pública & $\mathrm{x}$ & $x$ & $x$ & $x$ & $x$ \\
\hline & Accesibilidad & - & - & $x$ & - & - \\
\hline & Respeto a las costumbres & $\mathrm{x}$ & $x$ & $x$ & $x$ & - \\
\hline & Uso de materiales regionales & - & $x$ & - & $x$ & - \\
\hline & \begin{tabular}{|l|} 
Impacto visual \\
\end{tabular} & $=$ & $x$ & $\mathrm{x}$ & $\mathrm{x}$ & - \\
\hline & Funcionalidad y flexibilidad & - & $x$ & $\mathrm{x}$ & $x$ & $x$ \\
\hline \multirow{10}{*}{ Economía } & Consumo energético & - & $\mathrm{x}$ & $x$ & $x$ & $\mathrm{X}$ \\
\hline & Coste en el Ciclo de Vida & $\mathrm{x}$ & $\mathrm{x}$ & $x$ & $\mathrm{x}$ & $x$ \\
\hline & Uso de energías renovables & - & $x$ & $x$ & $x$ & $x$ \\
\hline & \begin{tabular}{|l} 
Relación Coste / Beneficio \\
\end{tabular} & $\mathrm{x}$ & $x$ & $x$ & $x$ & $x$ \\
\hline & $\begin{array}{l}\text { Adaptación y vulnerabilidad al } \\
\text { cambio climático }\end{array}$ & - & - & $x$ & - & - \\
\hline & \begin{tabular}{|l|} 
Diseño para el desmontaje \\
\end{tabular} & $\mathrm{x}$ & - & - & $\mathrm{x}$ & $\mathrm{x}$ \\
\hline & $\begin{array}{l}\text { Gobernabilidad, gestión del } \\
\text { proyecto }\end{array}$ & $x$ & $x$ & $x$ & $x$ & $x$ \\
\hline & Innovación en el proyecto & $x$ & $x$ & $\mathrm{x}$ & $x$ & $x$ \\
\hline & $\begin{array}{l}\text { Gastos ocasionados a los } \\
\text { usuarios }\end{array}$ & - & - & $\mathrm{x}$ & - & - \\
\hline & Incremento valor económico & - & - & $\mathrm{x}$ & - & - \\
\hline
\end{tabular}

Cabe destacar también que muchas de las variables ambientales empleadas (biodiversidad, efecto barrera, valor ecológico del suelo, paisaje, etc.) se han podido evaluar más fácilmente pues los proyectos están ya considerando estos criterios de algún modo debido a la obligatoriedad de la Evaluación de Impacto Ambiental. Sin embargo, otros indicadores considerados como emergentes, como son las emisiones de $\mathrm{CO}_{2-\mathrm{eq}}$, el consumo energético, el diseño pensando en el ciclo de vida o la aplicación de una gestión de riesgos como se hace en otros proyectos de ingeniería, todavía no se han interiorizado lo suficiente (o no existen herramientas operativas para su uso) de manera que puedan evaluarse desde las fases iniciales. Muchos de estos criterios sí que están comenzando a ser aplicados en etapas posteriores, proyecto de construcción o la ejecución en sí misma, como demuestra la actual obligación de un plan y estudio de gestión de residuos, la gestión de riesgos de autoprotección en determinadas infraestructuras (en especial los túneles), los estudios de seguridad y salud en la obra contemplando también a terceras personas, los costes en la fase de mantenimiento y explotación, etc. En la evaluación del caso de estudio expuesto, se ha tratado de valorar el impacto en el ciclo de vida de cada solución en cada criterio. Sin embargo, los proyectos actualmente no suelen disponer de información sobre la explotación y el mantenimiento de acuerdo a los distintos criterios aquí evaluados, y mucho menos de una posible demolición o deconstrucción. Es necesario, por tanto, un mayor desarrollo técnico de las alternativas en relación a su vida útil y no sólo de la etapa de construcción. Como se mostraba en la Tabla 3, no todas las etapas han podido ser evaluadas en el caso práctico en ocasiones por falta de información científica y en otras porque actualmente los proyectos en etapas tempranas no disponen de la información necesaria. Hasta entonces, los resultados y las evaluaciones de sostenibilidad serán posibles pero deberán manejarse los resultados con sumo cuidado teniendo en cuenta las limitaciones existentes y siendo siempre explícitos en las mismas.

En el caso analizado se observa que de aplicarse el sistema de indicadores propuesto en el primer tramo, la alternativa mejor valorada permitiría reducir los impactos sobre las dimensiones medioambiental, social y económica frente al resto de soluciones de una manera importante, obteniendo un índice cuatro veces superior a la alternativa 1 y veinte veces superior a la alternativa 2. En el resto de tramos, la importancia que se conceda a cada dimensión y criterio es fundamental para la selección de una u otra 
solución. Cuando las valoraciones de acuerdo a los distintos indicadores y dimensiones es muy similar, la sensibilidad de la valoración final de acuerdo a la asignación de pesos y al método multicriterio escogido es muy grande, por lo que la toma de decisiones debe realizarse con una intensa relación con el centro decisor. En cualquier caso, queda patente el largo camino que queda para asentar una evaluación integral y fiable de los impactos de sostenibilidad en los proyectos de ingeniería civil contemplando estos en su ciclo de vida.

\section{BIBLIOGRAFÍA}

(1) United Nations: Agenda 21: The United Nations Programme of Action From Rio. United Nations, New York, 1992.

(2) Bakens, W.: "Realizing the sector's potential for contributing to sustainable development". UNEP Industry and Environment, April-September (2003), pp. 9-12.

(3) Alarcón Núñez, D. B.: "Modelo Integrado deValor para Estructuras Sostenibles". Tesis doctoral, Universitat Politècnica de Catalunya, Escola Tècnica Superior D'Enginyers de Camins, Canals i Ports, 2005.

(4) Oteiza, I.; Tenorio, J. A.: "Jornada J7: Evaluación de la sostenibilidad en la Edificación". XVII Edición Curso de Estudios Mayores de la Construcción, La innovación en las técnicas, los sistemas y los materiales de construcción CEMCO. Madrid 7 de Junio, 2007.

(5) CRISP - Construction and City Related Sustainability Indicators: http://crisp.cstb.fr

(6) Fernández Sánchez, G.: "Análisis de los Sistemas de Indicadores de Sostenibilidad. Planificación urbana y proyectos de construcción". Escuela Técnica Superior de Ingenieros de Caminos, Canales y Puertos de Madrid, Universidad Politécnica de Madrid. Junio, 2008.

(7) Macías, M.; García Navarro, J.: "Metodología y herramienta VERDE para la evaluación de la sostenibilidad en edificios". Informes de la Construcción, vol. 62, no 517 (2010), pp. 87-100. doi: 10.3989/ ic.08.056

(8) Burgueño Muñoz, A.: "La evaluación de la sostenibilidad en obra civil". Conferencia V Congreso Nacional de Ingeniería Civil: Desarrollo y Sostenibilidad en el Marco de la Ingeniería. Sevilla, 26-28 de noviembre, 2007.

(9) Rodríguez Moya, J. A.: “Aportación metodológica para la Evaluación de la Sostenibilidad de Planes y Programas de Infraestructuras de Transportes. Aplicación al marco regional: Comunidad de Madrid". Tesis doctoral. Universidad Politécnica de Madrid, E.T.S. de Ingenieros de Caminos, Canales y Puertos. Madrid, 2005.

(10) Colegio de Ingenieros de Caminos, Canales y Puertos: Desarrollo y Sostenibilidad en el Marco de la Ingeniería. V Congreso Nacional de la Ingeniería Civil. Conclusiones. Ed. Colegio de Ingenieros de Caminos, Canales y Puertos. Madrid, 2009. ISBN: 978-84-380-0403-6.

(11) Ugwu, O. O.; Kumaraswamy, M. M.; Wong, A.; Ng, S. T.: "Sustainability appraisal in infrastructure projects (SUSAIP) Part 1. Development of indicators and computational methods". Automation in Construction, vol. 15 Issue 2 (2006), pp. 239-251. doi:10.1016/j.autcon.2005.05.006.

(12) Dasgupta, S.; Tam, EKL: "Indicators and framework for assessing sustainable infrastructure". Canadian Journal of Civil Engineering, vol. 32 Issue 1 (2005), pp. 30-44. doi: 10.1139/L04-101.

(13) Campbell, A. J.: "A multicriteria approach for rating roadway sustainability". Thesis. Department of Civil and Environmental Engineering, Florida State University, FAMU/FSU College of Engineering, 2009.

(14) Soderlund, M.: "Sustainable Roadway Design, A model for an environmental rating system". Thesis. Civil and Environmental engineering, University of Washington, 2007.

(15) Aguado, A. et al.: (Grupo de Trabajo Anejo ICES de la EHE). El índice de Contribución de las Estructuras a la Sostenibilidad (ICES). V Congreso Nacional de Ingeniería Civil: Desarrollo y Sostenibilidad en el Marco de la Ingeniería. Sevilla 26-28 de Noviembre, 2007.

(16) Campbell-Lendrum, E.; and Feris, J.: "Trialling Ceequal on a London railway embankment". Proceedings of the Institution of Civil Engineers, Engineering Sustainability, March (2008), pp. 71-76. doi: 10.1680/ensu.2008.161.1.71

(17) Fernández-Sánchez, G.; Rodríguez-López, F.: "A methodology to identify sustainability indicators in construction project management-Application to infrastructure projects in Spain". Ecological Indicators 10 (2010), pp. 1193-1201. doi:10.1016/j.ecolind.2010.04.009.

(18) ISO 21929-1. Sustainability in building construction-Sustainability indicators-Part 1 : Framework for development of indicators for buildings. International Organization for Standardization. March, 2006.

(19) Bell, S.; Morse, S.: Sustainability Indicators. Measuring the Immesurable Second edition. Earthscan. London, UK, 2008.

(20) Aguado, A.: Evaluación de la sostenibilidad en la EHE. Jornada "Construcción Sostenible: Nuevas perspectivas y Normalización". Instituto Español del Cemento y sus Aplicaciones (IECA). Madrid, 2010.

(21) Calderón, E. J.; Pronello, C.; Goger, T. (eds.): Integrated assessment of environmental impact of traffic and transport infrastructure. COST Action 350. Madrid, 2009.

(22) Gómez-Senent, E.; Chiner, M.; Chiner, M.J.: PRES : Programa de Evaluación de Proyectos Sociales. VII Congreso Nacional de Ingeniería de Proyectos. Zaragoza 26-28 Junio, 1991, pp. 27-35.

(23) Aragonés, P.: Aproximación a la Toma de Decisiones Multicriterio en Proyectos. Implementación de una metodología Multicriterio y Multiexperto: PRES II. Tesis doctoral. Universidad Politécnica de Valencia, 1997. 